\title{
Role of minimally invasive partial nephrectomy in the management of renal mass
}

\author{
Randall A. Lee, David Strauss, Alexander Kutikov \\ Division of Urology, Department of Surgery, Fox Chase Cancer Center, Temple University Health System, Philadelphia, PA, USA \\ Contributions: (I) Conception and design: A Kutikov; (II) Administrative support: A Kutikov; (III) Provision of study materials or patients: None; (IV) \\ Collection and assembly of data: RA Lee; (V) Data analysis and interpretation: RA Lee, D Strauss; (VI) Manuscript writing: All authors; (VII) Final \\ approval of manuscript: All authors. \\ Correspondence to: Randall A. Lee, MD. Division of Urology, Department of Surgery, Fox Chase Cancer Center, Temple University Health System, \\ 333 Cottman Avenue, Philadelphia, PA 19111, USA. Email: Randall.lee@tuhs.temple.edu.
}

\begin{abstract}
Partial nephrectomy is recommended for surgical management of small renal masses (SRM), or lesions $\leq 7 \mathrm{~cm}$. The decision for surgical intervention involves a balanced patient assessment. Minimally invasive approach, which includes laparoscopic and robotic techniques, has shown to have improved blood loss, length of hospitalization, and post-operative pain while maintaining oncologic efficacy when compared to an open approach. Transperitoneal approach is preferred at most centers; however, retroperitoneoscopic minimally invasive surgery (MIS) partial nephrectomy expertise is essential for comprehensive kidney cancer care. With advances in surgical technology and deep penetration of robotics into surgical training and practice, robotic partial nephrectomy has become the modality of choice in modern clinical practice. This review discusses the indications and outcomes for various minimally invasive approaches of partial nephrectomy.
\end{abstract}

Keywords: Minimally invasive; robotic; laparoscopic; partial nephrectomy

Submitted Aug 12, 2019. Accepted for publication Dec 10, 2019.

doi: $10.21037 /$ tau.2019.12.24

View this article at: http://dx.doi.org/10.21037/tau.2019.12.24

\section{Introduction}

The management of small renal masses (SRM, $<7 \mathrm{~cm}$ ) has evolved over the years. Oncologic safety and long-term benefits for partial nephrectomy over radical nephrectomy in appropriately selected patients is well-established (1-3). Current guidelines recommend nephron sparing surgery (NSS) for the management of SRM when technically feasible and most procedures are currently performed harnessing minimally invasive techniques (3). The minimally invasive surgical revolution began with the initial report of the laparoscopic radical nephrectomy in 1991 (4). Since then the once gold standard open approach transitioned to a pure laparoscopic approach. The minimally invasive surgery (MIS) techniques continued to evolve with the introduction and advancements in robotic surgery which lowered technical barriers inherent to laparoscopy. Here we review the minimally invasive approaches for the surgical management of renal masses.

\section{Preoperative evaluation: patient, tumor, and surgeon factors}

The incidence of renal masses has grown with the increased use of cross-sectional imaging (5). Each patient with renal mass must be carefully evaluated, as treatment decisions are nuanced and must be individualized. Patient selection for partial nephrectomy must integrate patient, tumor, and surgeon factors (Table 1) (6).

Anatomic tumor complexity must be understood and considered in advance renal surgery. Several scoring metrics to objectify reporting of the anatomic relationship between the tumor and the kidney have been described (7). The RENAL Nephrometry score (NS) is the first and 
Table 1 Pre-operative evaluation for partial nephrectomy encompassing patient, tumor, and surgeon factors [adapted from Lee et al. with permission (6)]

\begin{tabular}{|c|c|c|}
\hline Patient factors & Tumor factors & Surgeon factors \\
\hline Need for anticoagulation/ anti-platelet agents & Anatomic complexity (RENAL Nephrometry score) & - \\
\hline History of inflammatory bowel syndromes & Anterior vs. posterior location & - \\
\hline Perirenal fat and BMI & Proximity to hilum & - \\
\hline Patient preference & - & - \\
\hline
\end{tabular}

BMI, body mass index; RPN, registered practical nurse; LPN, licensed practical nurse.

the most commonly used such scoring system. The RENAL Score objectifies documentation and reporting of tumor size (R), endophytic or exophytic relationship to the renal parenchyma (E), proximity/nearness to the collecting system $(\mathrm{N})$, anterior or posterior position (A), and location relative to polar lines (L). Low complexity lesions scored are categorized as those with a score of $4-6$, intermediate complexity 7-9, and high complexity tumors are those with 10-12 score. Tumors abutting the main renal artery of vein receive an "h" suffix (8). While tumors with higher anatomic complexity are more likely to harbor high grade pathology, hilar location should not discourage nephron preservation (9). For instance, a recent report by Correa et al. reviewed 1,324 renal lesions and found no significant difference in higher nuclear grade $(39.8 \%$ vs. $34.3 \%)$, incidence of malignancy $(87.2 \%$ vs. $82.6 \%)$, or risk of upstaging between hilar and non-hilar masses. Extracapsular extension was identified more frequently in nonhilar masses. No difference was seen in renal sinus fat or vascular invasion $(\mathrm{P}=0.269)$ (10).

Various other anatomic scoring systems have been developed to help understand and predict outcomes following nephron-sparing surgery. The cortical surface area (CSA) quantifies the tumor surface area contiguous with benign parenchyma and has been shown to correlate with the preservation of renal function, LOS, and operative time (11). The arterial based complexity (ABC) scoring system categorizes renal masses based the vasculature dissected and/or transected during PN (12). Careful evaluation of the retroperitoneum should include the perinephric and peri-sinus fat for presence of T3a disease.
Patients with elevated BMI and perinephric fat thickness can harbor "toxic" sticky fat, increasing dissection difficulty, OR time, and complication rate $(13,14)$.

The role of renal mass biopsy (RMB) in the management of renal masses is expanding. Although every patient may not benefit from renal biopsy, its appropriate use may prevent overtreatment $(3,15)$. Currently, guidelines recommend a utility-based approach. RMB are not indicated for individuals where results are unlikely to alter management. For instance, biopsies may be non-actionable in younger patients who are unwilling to undergo surveillance for masses that may yield indolent biopsy results; meanwhile, elderly and frail patients who are poor candidates for intervention regardless of what pathology from a biopsy also may not need to undergo the procedure. Nevertheless, many patients can benefit from appropriate calibration of care intensity based on renal biopsy results, especially given overall safety of the procedure $(3,15,16)$.

\section{Intraoperative considerations}

Kidney is an extremely vascular organ and cessation of vascular flow to the organ is often necessary in order to achieve appropriate visualization and safe resection. Ischemia strategies can be placed into three general categories: (I) cold ischemia time (CIT), (II) warm ischemia time (WIT), and (III) zero ischemia time (ZIT). Hypothermia from CIT limits post ischemic renal injury by halting renal metabolism (17). Nevertheless, WIT is the most frequently-employed strategy, especially during MIS techniques. The human kidney tolerates warm ischemia 
extremely well and, although WIT times are generally minimized, there are no robust data nor expert consensus for safe WIT limits (17-19). After some initial enthusiasm for selective arterial clamping and/or off clamp NSS, these techniques are not utilized ubiquitously and appear to achieve results indistinguishable from those of WIT $(3,19,20)$.

When performing NSS, complete tumor resection is imperative. Resection strategies can be categorized as simple enucleation, enucleoresection, or wedge resection; however, there is currently no evidence supporting one universal technique (21). It is recommended that tumor enucleation be performed for patients with familial renal cell carcinoma, multifocal disease, or severe CKD in order to maximally preserve renal parenchyma (3). SurfaceIntermediate-Base (SIB) margin score was introduced in 2014 to help standardize reporting of resection techniques (21). SIB objectifies documentation of the amount of renal parenchyma that covers the tumor after resection employing visual inspection. Fidelity of this visual inspection has been validated by its comparison with histologic findings at pathology (21). At our institution, we generally attempt to leave a minimal layer of renal tissue on the tumor (SIB score 1/1/1) in order to preserve tumor integrity and avoid positive margins; nevertheless, it is not infrequent to enucleate the tumor at its based in order to facilitate resection safety. Intraoperative imaging techniques may be used to aid resection. Intraoperative ultrasound provides real time imaging of tumor localization, tumor border delineation, and confirmation of appropriate ischemia. While intraoperative use of fluorescent imaging can help aid dissection with the identification of renal vasculature, the benefits of fluorescent imaging are debated.

Once the tumor is resected, integrity of the vasculature and the collecting system must be restored, while renal parenchyma is generally reapproximated. Numerous techniques have been described; however, there is no consensus on superiority of one $v s$. another strategy (22). At our institution, the tumor base is re-approximated with a 3-0 absorbable barbed suture. Depending on size of the resection, overlapping suture lines may be used. The renal parenchyma is re-approximated with absorbable barbed 2-0 suture in a horizontal mattress fashion. The use of hemostatic agents within the renorrhaphy bed is limited, however loose knit oxidized cellulose bolsters constructed with absorbable suture are sometimes used to fill expected dead space within the closure. Appropriate tissue tension is achieved using sliding clip technique on the parenchymal side of the closure. Early unclamping prior to the completion of renorrhaphy can help identify exposed vessels at the tumor base that were not ligated during the initial reapproximation. A drain is generally only be left when the integrity of the renorrhaphy repair is in question as recent evidence show surgical drains may not be necessary in the majority of cases (Figure 1) $(23,24)$.

Regardless of surgical technique and approach, older patients ( $>65$ years old) and those with high complexity renal masses undergoing partial nephrectomy experienced higher rates of post-operative complications $(25,26)$. Choice between partial and radical nephrectomy can be challenging and expected eGFR following complete renal unit removal may drive critical clinical decision-making. Indeed, AUA Guidelines suggest that when eGFR is expected to drop $<45 \mathrm{~mL} / \mathrm{min} / 1.73 \mathrm{~m}^{2}$ (27). Recently, a predictive model to help anticipate postoperative renal function decline below this threshold was reported (28).

\section{Open versus laparoscopic partial nephrectomy}

Since the introduction of laparoscopy for renal surgery in 1991, perioperative outcomes of open $v$ s. minimallyinvasive partial nephrectomy (LPN) has been extensively documented. One of the most robust early comparisons was performed by Gill et al. This seminal work evaluated 1,800 patients who underwent either laparoscopic or open partial nephrectomy, demonstrating LPN as a safe and effective alternative to open partial nephrectomy (OPN) (29,30). A more recent study by Springer et al. compared 170 OPN cases and $170 \mathrm{LPN}$ cases, demonstrated a significant reduction in WIT for both OPN and LPN in this more modern cohort (11.7 min OPN and $14.4 \mathrm{~min}$ LPN vs. $30.7 \mathrm{~min}$ LPN and $20.1 \mathrm{~min}$ OPN) (31). Limitations included differences in tumor characteristics between groups in these preselected cohorts. As expected LPN was shown to have significant lower analgesic requirement, 20.2 vs. $252.5 \mathrm{mg}$ morphine, and both studies noted a significantly shorter length of hospitalization, 2 vs. 5 days for patients undergoing minimally-invasive surgery $(30,32)$. Cancer specific survival (CSS) appears uncompromised with MIS (32). When compared at 5 year follow up, CSS and overall survival for LPN were reported to be $94 \%$ and $91 \%$ respectively with similar outcomes for open technique of $92 \%$ and $88 \%$, respectively $(31,33)$. In 2013 , Lane et al. performed a retrospective study on 1,541 patients with 
Perioperative considerations for minimally invasive partial nephrectomy

Preoperative evaluation

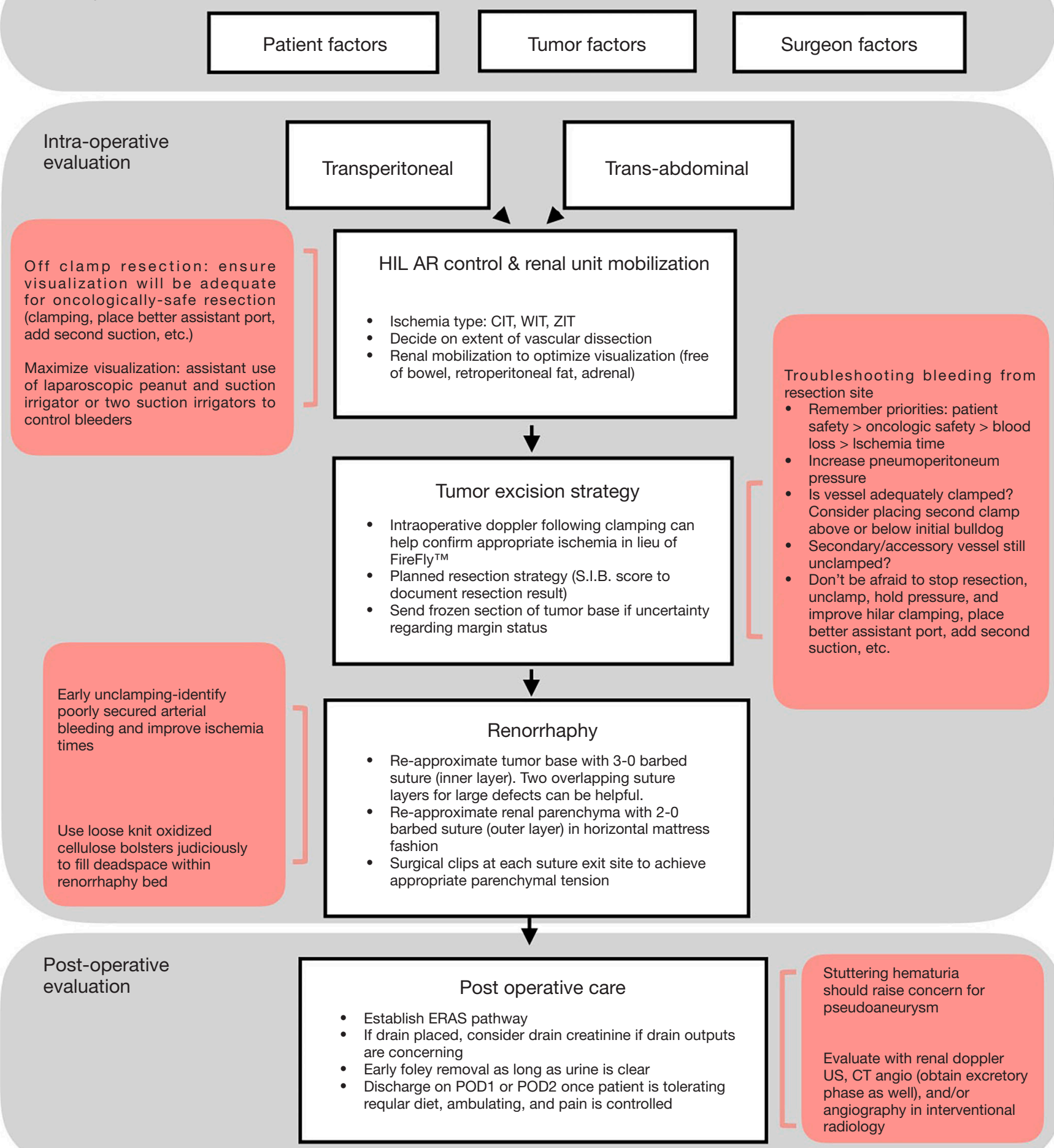

Figure 1 Perioperative evaluation for minimally invasive partial nephrectomy. Flow chart highlighting preoperative, intraoperative, and post-operative considerations for minimally invasive partial nephrectomy. Pre-operative factors are further discussed in Table 1. Red boxes summarize commonly encountered complications and discussion points during each corresponding step. Adapted from Lee et al. with permission (6). 
$\leq \mathrm{cT} 1$ lesions undergoing LPN or OPN and reported 10-year oncologic outcomes. Results demonstrated a metastasis-free survival rate on $95.2 \%$ in the laparoscopic cohort and $90.0 \%$ in the open cohort, with no significant differences noted in reduction in median GFR between groups. Selection bias for more complex, and thus more biologically aggressive masses, undergoing open partial nephrectomy likely accounts for the difference in oncologic outcomes. In fact, in a multivariate analysis for all-cause mortality operative approach was not a significant predictor of outcome, concluding that the OSS at 10 years was mediated by patient factors, not operative technique (34). Thus, when compared to open surgery, the laparoscopic approach provides equivalent outcomes with added benefits of improved post-operative pain and reduction in hospitalization.

\section{Robotic partial nephrectomy}

Although the laparoscopic approach for NSS gained popularity, the technical challenges and the steep learning curves associated with these techniques kept limited the number of surgeon comfortable with LPN to a small group of experts (32). In fact, NSS was felt to be underutilized and laparoscopic radical nephrectomy overused, before the robotic platform gained traction in the kidney cancer space (35). The introduction of robotics lowered technical barriers and expanded on the capabilities of minimally invasive NSS. The three-dimensional magnified optics and increased dexterity allowed contoured resection and easier renorrhaphy suturing (36). With the almost universal incorporation of robotics in surgical training and shorter learning curve, robotic approach rapidly became the firstline treatment modality for NSS $(37,38)$.

Multiple large retrospective analyses have evaluated outcomes following licensed practical nurse (LPN) vs. registered practical nurse (RPN). RPN has shown to have significantly lower EBL, LOS, and WIT $(39,40)$. Metaanalyses demonstrate the transition away from laparoscopic approach resulted in a case-mix involving lesions with higher anatomic complexity (6). RPN were shown to have decreased likelihood of conversion to open, minor and major complications, and positive surgical margins (39). Majority of studies comparing the two MIS approaches have shown a shorter WIT with RPN (40-43).

In 2012, Simhan et al. sought to compare the robotic approach to the gold standard OPN for moderate (NS 7-9) and highly (NS 10-12) complex renal masses. Comparison of cohorts showed that lesions undergoing OPN were larger in size based on pre-operative imaging $(3.9$ vs. $3.0 \mathrm{~cm}$, $\mathrm{P}<0.001)$, but noted no differences in demographics or Charlston comorbidity index (CCI). The open cohort for both moderately and highly complex lesions were noted to have high pathologic stage. The robotic cohort had similar outcomes with significantly lower EBL and LOS. More recently in 2018, Garisto et al. retrospectively compared 203 RPN and 76 OPN for complex lesions with a median RENAL Nephrometry score of 10 (44). Results concurred with findings of Simhan et al., noting significant differences favoring RPN in EBL, intraoperative transfusion rates, and length of hospitalizations. Results further supported the implementation of robotics for complex tumors with no statistical differences in positive surgical margins (RPN $10 \%$ and OPN 14.9\%) (44). Differences in sample sizes between RPN and OPN between the two studies (Simhan et al.: 91 vs. 190, Garisto et al.: 203 vs. 76) represent the overall improvements in surgeon familiarity and comfort level with robotic surgery.

\section{Trans-abdominal versus retroperitoneoscopic robotic partial nephrectomy}

With advancements in robotic surgery, the robotic approach has become the preferred option for both patients and surgeons. Trans-abdominal robotic approach has been supplemented with utilization of retroperitoneoscopic robotic techniques. This "retro" approach allows MIS for patients with history of extensive intraperitoneal scarring, since transperitoneal dissection is entirely avoided. It also provides direct access to tumors on the posteriorly aspect of the kidney, especially those behind the renal hilum, making renal unit rotation unnecessary (Figure 1).

In the trans-abdominal approach, patient is first placed in the modified lateral decubitus position, with the affected kidney up and the table placed in full flexion. Pneumoperitoneum is achieved using a Veress needle. A $12-\mathrm{mm}$ camera port is first placed at the level of the renal hilum at the mid-clavicular line. Eight mm robotic ports are placed along the ipsilateral midclavicular line with the superior port placed 1-2 finger breaths off the inferior costal margin. Next, a $12-\mathrm{mm}$ and $5-\mathrm{mm}$ assistant ports are placed toward the midline. A $3^{\text {rd }} 8 \mathrm{~mm}$ port is placed $1-2$ finger breaths off the inferior costal margin as needed. At least $7 \mathrm{~cm}$ should be placed between each port (Figure 2). 

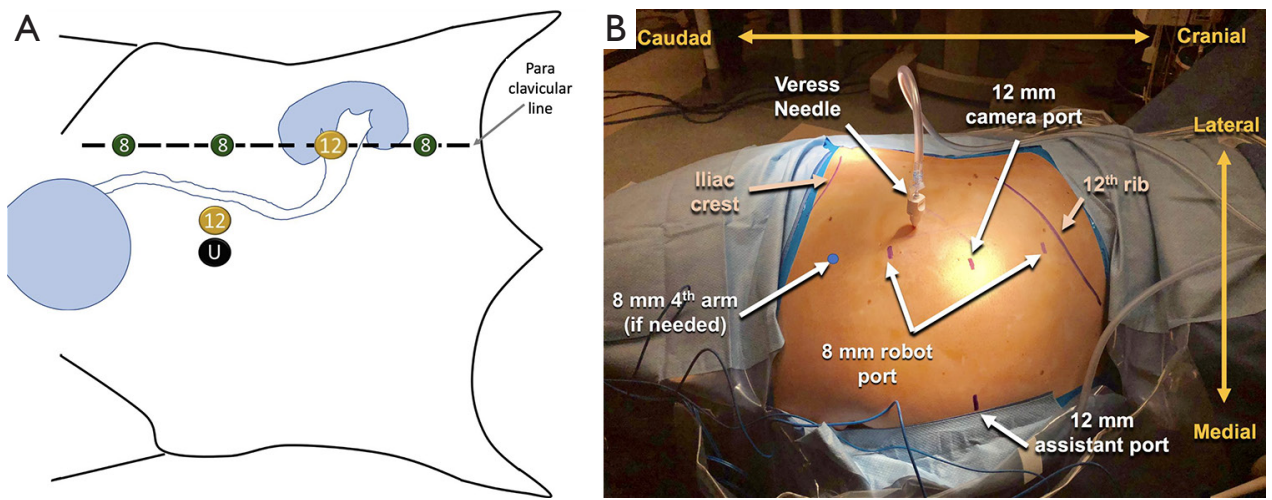

Figure 2 Port placement for transperitoneal robotic partial nephrectomy. (A) Diagram showing port placement along the mid-clavicular line; (B) location of anatomical landmarks and ports marked following positioning and achievement of the pneumoperitoneum. Fourth arm port is marked and placed on a case by case basis.

Once ports are placed and the robot is docked, the procedure begins with the identification and dissection of the plane between the colon and Gerota's fascia. Dissection is continued until identification of the ureter, which can be seen coursing adjacent to the gonadal vessels. The ureter and lower pole of kidney can be lifted to optimize exposure and dissection of the hilum. Once vessels are carefully dissected, the kidney is mobilized as necessary and separated from surrounding adipose tissue. Once the plan for resection is in place, the renal vasculature is clamped. In most cases, only clamping of the renal artery is necessary, since the pneumoperitoneum largely tamponades venous bleeding. Following resection, renorrhaphy is performed as described above.

Paucity of anatomic landmarks and lack of familiarity with this technique during surgical training has made retroperitoneoscopic approach less frequently utilized. Positioning for retroperitoneal approach is similar to transabdominal approach; however, appropriate flexion is especially critical. The operative table is flexed to increase distance between the iliac crest and the subcostal margin. $12 \mathrm{~mm}$ camera port site is marked in the posterior axillary line between the tip of the 12 th rib and the iliac crest. A lateral $8 \mathrm{~mm}$ port site is marked $6-8 \mathrm{~cm}$ from the $12-\mathrm{mm}$ camera port and medial $8 \mathrm{~mm}$ ports are marked $6-8 \mathrm{~cm}$ from the $12-\mathrm{mm}$ camera port. The assistant $12 \mathrm{~mm}$ port site marked just off the iliac crest and triangulated between the $12-\mathrm{mm}$ camera port and the first medial $8 \mathrm{~mm}$ robotic port (Figure 3).

First, the $12-\mathrm{mm}$ camera port incision is made. Using blunt dissection down to the lumbodorsal fascia, the RP space is entered with blunt instrument and the space is developed with finger dissection. The trocar balloon dilator is placed into the developed space and insufflated (40 pumps) to further develop the retroperitoneal working area behind the kidney. An $8-\mathrm{mm}$ trocar is placed $8 \mathrm{~mm}$ lateral to the initial camera port. A balloon-tipped port is placed in the original incision and a laparoscopic Kittner is harnessed to sweep the peritoneum medially. Generally, a 30-degree up camera is employed. Remaining ports are placed under direct vision and the robot is docked. It is helpful to dock one arm at a time before docking the camera itself in order to facilitate visualization and advancement of the arms into the appropriate locations. Once the robot is docked, Gerota's fascia is incised longitudinally. Medial and anterior tension on the renal unit facilitates tension on the hilum. When operating on the right renal unit, the renal artery is readily found behind the vena cava by following the psoas muscle. On the left, the psoas muscle is followed to the paraaortic lymph nodes, and the renal artery is generally identified anterior to these nodes. Once the vasculature and renal mass are fully dissected, the renal artery is controlled. Excision and renorrhaphy is performed as described above.

\section{Conclusions}

In summary, renal surgery remains the gold standard treatment for patients with renal mass. Regardless of approach or technique, patient and oncologic safety must be the top clinical priority. NSS should be utilized wisely and combined with minimally invasive surgical techniques without compromise to long-term patient outcomes. 

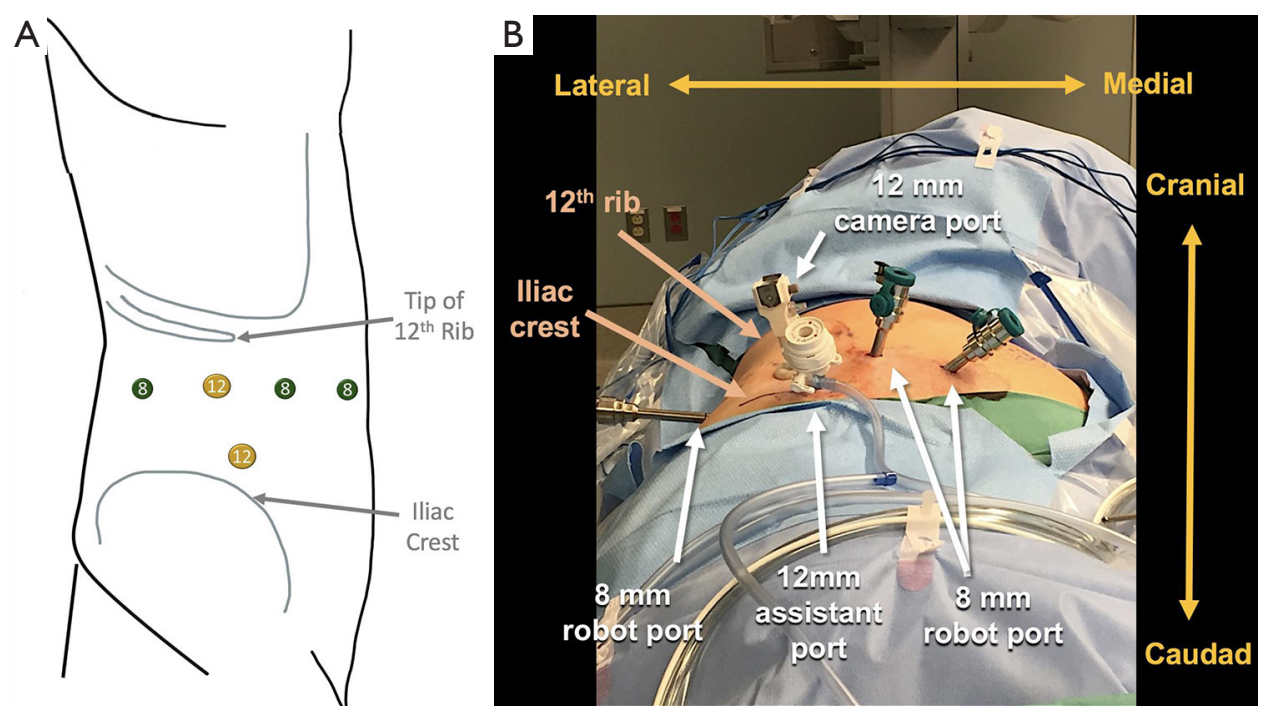

Figure 3 Port placement for retroperitoneoscopic robotic partial nephrectomy. (A) Diagram showing port placement; (B) placement of ports following positioning, development of retroperitoneal space, and insufflation of the retroperitoneum.

\section{Acknowledgments}

Funding: None.

\section{Footnote}

Provenance and Peer Review: This article was commissioned by the Guest Editor (Shomik Sengupta) for the series "Surgery for Urologic Cancers" published in Translational Andrology and Urology. The article has undergone external peer review.

Conflicts of Interest: All authors have completed the ICMJE uniform disclosure form (available at http://dx.doi. org/10.21037/tau.2019.12.24). The series "Surgery for Urologic Cancers" was commissioned by the editorial office without any funding or sponsorship. The authors have no other conflicts of interest to declare.

Ethical Statement: The authors are accountable for all aspects of the work in ensuring that questions related to the accuracy or integrity of any part of the work are appropriately investigated and resolved.

Open Access Statement: This is an Open Access article distributed in accordance with the Creative Commons Attribution-NonCommercial-NoDerivs 4.0 International License (CC BY-NC-ND 4.0), which permits the non- commercial replication and distribution of the article with the strict proviso that no changes or edits are made and the original work is properly cited (including links to both the formal publication through the relevant DOI and the license). See: https://creativecommons.org/licenses/by-nc-nd/4.0/.

\section{References}

1. Gu L, Ma X, Li H, et al. Comparison of oncologic outcomes between partial and radical nephrectomy for localized renal cell carcinoma: A systematic review and meta-analysis. Surg Oncol 2016;25:385-93.

2. Van Poppel H, Da Pozzo L, Albrecht W, et al. A prospective randomized EORTC intergroup phase 3 study comparing the complications of elective nephron-sparing surgery and radical nephrectomy for low-stage renal cell carcinoma. Eur Urol 2007;51:1606-15.

3. Campbell S, Uzzo RG, Allaf ME, et al. Renal Mass and Localized Renal Cancer: AUA Guideline. J Urol 2017;198:520-9.

4. Clayman RV, Kavoussi LR, Figenshau RS, et al. Laparoscopic nephroureterectomy: initial clinical case report. J Laparoendosc Surg 1991;1:343-9.

5. Smaldone MC, Egleston B, Hollingsworth JM, et al. Understanding Treatment Disconnect and Mortality Trends in Renal Cell Carcinoma Using Tumor Registry Data. Med Care 2017;55:398-404.

6. Lee RA, Kutikov A, Uzzo RG. Robotic Nephron Sparing 
Surgery for High Complexity Renal Tumors. AUANews

2019. Available online: https://www.auanet.org/ publications/auanews/auanews-online-access

7. Srivastava A, Ficarra V, Kutikov A. The Alphabet Soup of Modern Nephrometry Systems. Eur Urol Oncol 2018;1:435-6.

8. Kutikov A, Uzzo RG. The R.E.N.A.L. nephrometry score: a comprehensive standardized system for quantitating renal tumor size, location and depth. J Urol 2009;182:844-53.

9. Kutikov A, Smaldone MC, Egleston BL, et al. Anatomic features of enhancing renal masses predict malignant and high-grade pathology: a preoperative nomogram using the RENAL Nephrometry score. Eur Urol 2011;60:241-8.

10. Correa AF, Yankey H, Li T, et al. Renal Hilar Lesions: Biological Implications for Complex Partial Nephrectomy. Urology 2019;123:174-80.

11. Leslie S, Gill IS, de Castro Abreu AL, et al. Renal tumor contact surface area: a novel parameter for predicting complexity and outcomes of partial nephrectomy. Eur Urol 2014;66:884-93.

12. Spaliviero M, Poon BY, Karlo CA, et al. An Arterial Based Complexity (ABC) Scoring System to Assess the Morbidity Profile of Partial Nephrectomy. Eur Urol 2016;69:72-9.

13. Joshi SS, Uzzo RG. Renal Tumor Anatomic Complexity: Clinical Implications for Urologists. Urol Clin North Am 2017;44:179-87.

14. Davidiuk AJ, Parker AS, Thomas CS, et al. Mayo adhesive probability score: an accurate image-based scoring system to predict adherent perinephric fat in partial nephrectomy. Eur Urol 2014;66:1165-71.

15. Kutikov A, Smaldone MC, Uzzo RG, et al. Renal Mass Biopsy: Always, Sometimes, or Never? Eur Urol 2016;70:403-6.

16. Ahmad AE, Kutikov A, Finelli A. Needle Tract Seeding Following Renal Tumor Biopsy: Scarcely a Concern or a Concern to Scare? Eur Urol 2019;75:868-70.

17. Gill IS, Abreu SC, Desai MM, et al. Laparoscopic ice slush renal hypothermia for partial nephrectomy: the initial experience. J Urol 2003;170:52-6.

18. Volpe A, Blute ML, Ficarra V, et al. Renal Ischemia and Function After Partial Nephrectomy: A Collaborative Review of the Literature. Eur Urol 2015;68:61-74.

19. Greco F, Autorino R, Altieri V, et al. Ischemia Techniques in Nephron-sparing Surgery: A Systematic Review and Meta-Analysis of Surgical, Oncological, and Functional Outcomes. Eur Urol 2019;75:477-91.

20. Antonelli A, Cindolo L, Sandri M, et al. Safety of on- vs. off-clamp robotic partial nephrectomy: per-protocol analysis from the data of the CLOCK randomized trial. World J Urol 2020;38:1101-8.

21. Minervini A, Carini M, Uzzo RG, et al. Standardized reporting of resection technique during nephron-sparing surgery: the surface-intermediate-base margin score. Eur Urol 2014;66:803-5.

22. Bahler CD, Sundaram CP. Effect of Renal Reconstruction on Renal Function After Partial Nephrectomy. J Endourol 2016;30 Suppl 1:S37-41.

23. Kriegmair MC, Mandel P, Krombach P, et al. Drain placement can safely be omitted for open partial nephrectomy: Results from a prospective randomized trial. Int J Urol 2016;23:390-4.

24. Kim SP, Campbell SC, Gill I, et al. Collaborative Review of Risk Benefit Trade-offs Between Partial and Radical Nephrectomy in the Management of Anatomically Complex Renal Masses. Eur Urol 2017;72:64-75.

25. Hennus PM, Kroeze SG, Bosch JL, et al. Impact of comorbidity on complications after nephrectomy: use of the Clavien Classification of Surgical Complications. BJU Int 2012;110:682-7.

26. Simhan J, Smaldone MC, Tsai KJ, et al. Objective measures of renal mass anatomic complexity predict rates of major complications following partial nephrectomy. Eur Urol 2011;60:724-30.

27. Brito J 3rd, Pereira J, Moreira DM, et al. The association of lymph node dissection with 30-day perioperative morbidity among men undergoing minimally invasive radical prostatectomy: analysis of the National Surgical Quality Improvement Program (NSQIP). Prostate Cancer Prostatic Dis 2018;21:245-51.

28. McIntosh AG, Parker DC, Egleston BL, et al. Prediction of significant estimated glomerular filtration rate decline after renal unit removal to aid in the clinical choice between radical and partial nephrectomy in patients with a renal mass and normal renal function. BJU Int 2019;124:999-1005.

29. Pereira J, Renzulli J, 2nd, Pareek G, et al. Perioperative Morbidity of Open Versus Minimally Invasive Partial Nephrectomy: A Contemporary Analysis of the National Surgical Quality Improvement Program. J Endourol 2018;32:116-23.

30. Gill IS, Matin SF, Desai MM, et al. Comparative analysis of laparoscopic versus open partial nephrectomy for renal tumors in 200 patients. J Urol 2003;170:64-8.

31. Springer C, Hoda MR, Fajkovic H, et al. Laparoscopic vs. open partial nephrectomy for T1 renal tumours: evaluation 
of long-term oncological and functional outcomes in 340 patients. BJU Int 2013;111:281-8.

32. Gill IS, Kavoussi LR, Lane BR, et al. Comparison of 1,800 laparoscopic and open partial nephrectomies for single renal tumors. J Urol 2007;178:41-6.

33. Marszalek M, Meixl H, Polajnar M, et al. Laparoscopic and open partial nephrectomy: a matched-pair comparison of 200 patients. Eur Urol 2009;55:1171-8.

34. Lane BR, Campbell SC, Gill IS. 10-year oncologic outcomes after laparoscopic and open partial nephrectomy. J Urol 2013;190:44-9.

35. Smaldone MC, Kutikov A, Egleston B, et al. Assessing performance trends in laparoscopic nephrectomy and nephron-sparing surgery for localized renal tumors. Urology 2012;80:286-91.

36. Maurice MJ, Ramirez D, KaoukJH. Advances in roboticassisted treatments for renal cell carcinoma. Curr Opin Urol 2016;26:417-23.

37. Mottrie A, De Naeyer G, Schatteman P, et al. Impact of the learning curve on perioperative outcomes in patients who underwent robotic partial nephrectomy for parenchymal renal tumours. Eur Urol 2010;58:127-32.

38. Patel HD, Mullins JK, Pierorazio PM, et al. Trends in renal surgery: robotic technology is associated with increased use of partial nephrectomy. J Urol

Cite this article as: Lee RA, Strauss D, Kutikov A. Role of minimally invasive partial nephrectomy in the management of renal mass. Transl Androl Urol 2020;9(6):3140-3148. doi: $10.21037 /$ tau.2019.12.24
2013;189:1229-35.

39. Choi JE, You JH, Kim DK, et al. Comparison of perioperative outcomes between robotic and laparoscopic partial nephrectomy: a systematic review and metaanalysis. Eur Urol 2015;67:891-901.

40. Leow JJ, Heah NH, Chang SL, et al. Outcomes of Robotic versus Laparoscopic Partial Nephrectomy: an Updated Meta-Analysis of 4,919 Patients. J Urol 2016;196:1371-7.

41. Kizilay F, Turna B, Apaydin E, et al. Comparison of long-term outcomes of laparoscopic and robot-assisted laparoscopic partial nephrectomy. Kaohsiung J Med Sci 2019;35:238-43.

42. Benway BM, Bhayani SB, Rogers CG, et al. Robot assisted partial nephrectomy versus laparoscopic partial nephrectomy for renal tumors: a multiinstitutional analysis of perioperative outcomes. J Urol 2009;182:866-72.

43. Wang AJ, Bhayani SB. Robotic partial nephrectomy versus laparoscopic partial nephrectomy for renal cell carcinoma: single-surgeon analysis of $>100$ consecutive procedures. Urology 2009;73:306-10.

44. Garisto J, Bertolo R, Dagenais J, et al. Robotic versus open partial nephrectomy for highly complex renal masses: Comparison of perioperative, functional, and oncological outcomes. Urol Oncol 2018;36:471.e1-9. 